\title{
Intravenous Fracture of a Peripheral Cannula at the Dorsum of the Hand in a Patient Who Used Walking Aids after Surgery
}

\author{
Chandana Karunathilaka* ${ }^{(0)}$, Vidura Wijesundara, Nalin Madushanka \\ University Hospital, General Sir John Kotelawala Defence University, Werahara, Sri Lanka \\ Email: *chandana375@hotmail.co.uk, vidhurawije@yahoo.com,nnnmadushanka@gmail.com
}

How to cite this paper: Karunathilaka, C., Wijesundara, V. and Madushanka, N. (2021) Intravenous Fracture of a Peripheral Cannula at the Dorsum of the Hand in a Patient Who Used Walking Aids after Surgery. Open Journal of Orthopedics, 11, 146-152.

https://doi.org/10.4236/ojo.2021.114014

Received: March 14, 2021

Accepted: April 24, 2021

Published: April 27, 2021

Copyright $\odot 2021$ by author(s) and Scientific Research Publishing Inc. This work is licensed under the Creative Commons Attribution International License (CC BY 4.0).

http://creativecommons.org/licenses/by/4.0/

\begin{abstract}
In orthopaedic patients, peripheral intravenous (IV) cannulation is a common procedure for various clinical purposes. This patient was introduced with a $17 \mathrm{G}$ cannula in the basilic vein of the dorsal venous arch of the left hand prior to knee replacement surgery. Post knee surgery patients use walking aids for mobilization. Cannula which has been placed at the dorsum of the hand has a potential to bend at the neck of the cannula when the wrist bend while holding the walking aid. Repeated bending can result in fatigue fracture of the cannula neck. In this patient at the time of cannula removal, it was noted the catheter part is broken and proximal migration. Ultrasound guided localization was done and removed with a venotomy under local anesthesia. It is advisable to place peripheral venous cannulas well away from the wrist joint, which will prevent catheter bending and fracture. This is a very important point to consider when placing cannulas in orthopaedic patients who undergo surgical procedures.
\end{abstract}

\section{Keywords}

Peripheral Intravenous (IV) Cannulation, Cannula Fracture,

Proximal Embolization

\section{Introduction}

In orthopaedic patients, peripheral intravenous (IV) cannulation is the easiest and most common procedure used to obtain intravenous access.

Peripheral intravenous cannulation is essential for the delivery of intravenous medications, fluids and other intravascular replacements. The procedure is usually performed by doctors, nurses and paramedical staff. The preferred placement for 
the IV cannula is the dorsal venous arch of the hand. IV cannulation is associated with several complications. Commonly documented complications are damage to the veins, arteries, or nerves; haematoma formation; mechanical, chemical, or septic phlebitis; cannula-related infections; and extravasation of medications.

Intravenous fracture of a cannula is rare, but it can occur and may result in serious consequences, such as distal embolization.

Most orthopaedic patients need walking aids (e.g., walking frames, crutches) for mobilization after surgery to distribute their body weight or keep weight off an operated leg. When using a walking aid, the wrist is bent to hold the grip of the walking aid. Most cannulas are placed in the dorsum of the hand or wrist since the dorsal venous arch is the most comfortable place to site a cannula. However, when an IV cannula is placed at the dorsum of the hand or wrist, the cannula has the potential to bend at the neck of the cannula when the wrist bend. Because of the frequent bending at the cannula neck, the catheter part of the cannula may fracture. Cannula fragments can be distally embolized and result in hazardous consequences.

In this case report, we discuss a case of an intravenous fracture of an IV cannula that was placed at the basilic vein of the dorsal venous arch of the hand. The patient underwent total knee replacement surgery. Postoperatively, the patient was mobilized with a walker, and during the mobilization procedure, the cannula was bent and fractured. The cannula acted as an intravascular foreign body and migrated proximally.

\section{Case Presentation}

A 65-year-old, female patient was admitted to the University Hospital of General Sir John Kotelawala Defence University, Sri Lanka for a right knee replacement. She was admitted after conducting all preoperative preparations for right knee replacement surgery. Her vital signs were stable, and all preoperative blood investigations were normal. Routine procedure for knee replacement surgery was followed. On the day of surgery, in the operation theatre, the consultant anaesthetist placed a $17 \mathrm{G}$ cannula in the basilic vein of the dorsal venous arch of the left hand (Figure 1).

The patient underwent a successful total right knee replacement. As a routine practice, she was given IV antibiotics for three days and routine IV fluid replacement. She started supported weight bearing and mobilization with a walking frame on postoperative day one. On postoperative day two, she was transfused with one pint of blood due to a low haemoglobin level $(9.1 \mathrm{~g} / \mathrm{dl})$.

Patients, postoperative recovery during first threedays proceeded smoothly without any complications. On the evening of postoperative day three, her IV cannula was not functioning. She was scheduled to be discharged on day four. Therefore, there was no requirement for further IV antibiotics and fluids, a decision to complete the rest of the antibiotic dose with oral antibiotics were taken. 


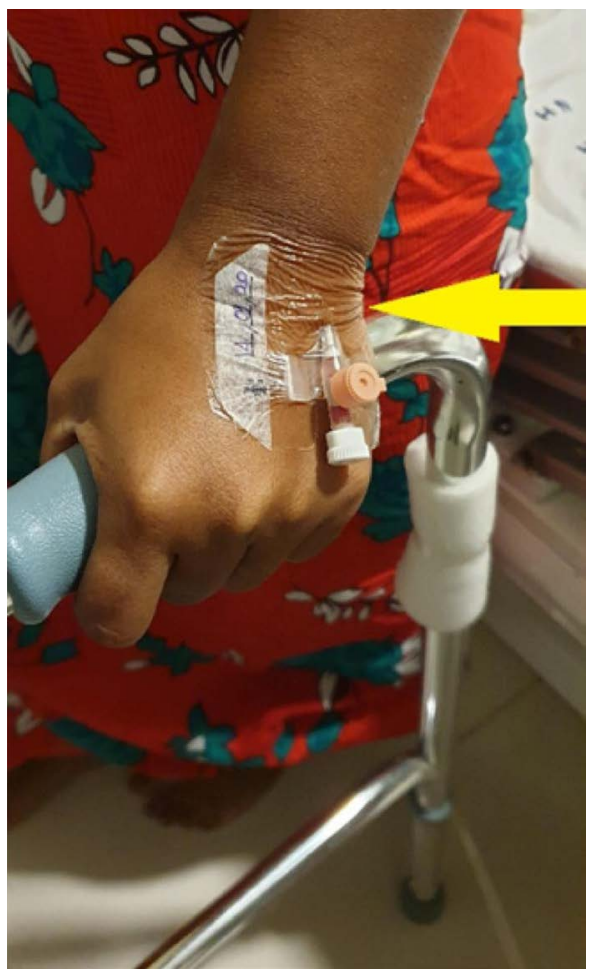

Figure 1. Cannula in the dorsal venous arch.

As discharge was planned on day four, the ward nurse had removed the cannula, and they immediately recognized portion of the intravenous cannula was missing. The IV cannula had broken at the neck of the cannula, and the nurse was unable to remove the stem of the cannula (Figure 2).

This was reported immediately, and instructions were given to apply a tourniquet in the proximal forearm near the elbow. The patient was taken to the operation theatre instantly, and an ultrasound scan (USS) was performed using a bed-side scanner (Figure 3 ).

The USS noted that the broken piece of the cannula was in the dorsal vein just $3.0 \mathrm{~cm}$ proximal to the point of insertion. A new tourniquet tube was applied closer to the proximal end of the broken cannula fragment.

Thepatients' forearm was cleaned and draped for surgery, the vein was explored under local anaesthesia, transverse venotomy was performed, and the broken cannula fragment was removed with curved mosquito forceps without any hazardous outcomes (Figure 4).

Both the proximal and distal ends of the vein were milked to remove the formed blood clots. The vein was ligated, and the skin was closed with 3.0 Ethilon suture. The patient made an uneventful recovery and was discharged with a follow-up plan for post-total knee arthroplasty.

The patient was seen again on postoperative day twelve for arthroplasty follow-up, and skin sutures of the venotomy side were removed. The skin healed nicely, and there was no evidence of thrombophlebitis of the veins and no local inflammation. 


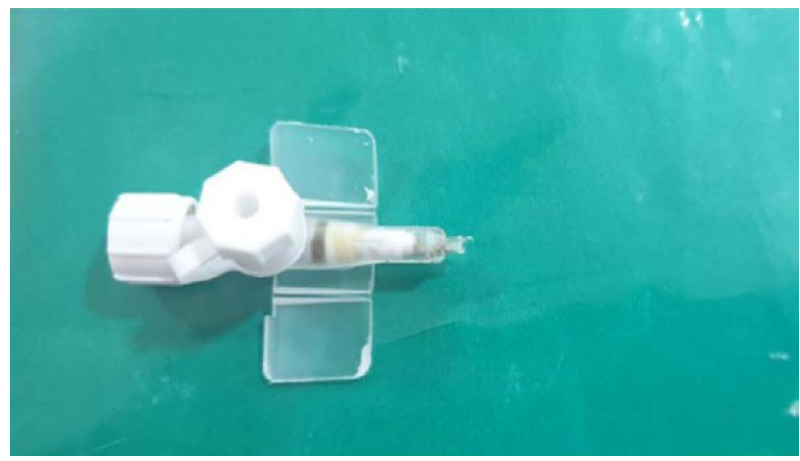

Figure 2. Broken cannula.

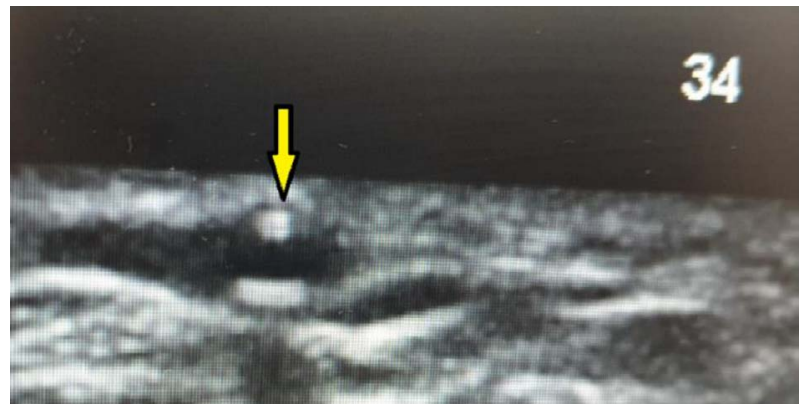

Figure 3. Ultrasound scan: yellow arrow indicates the intravascular portion of the broken cannula.

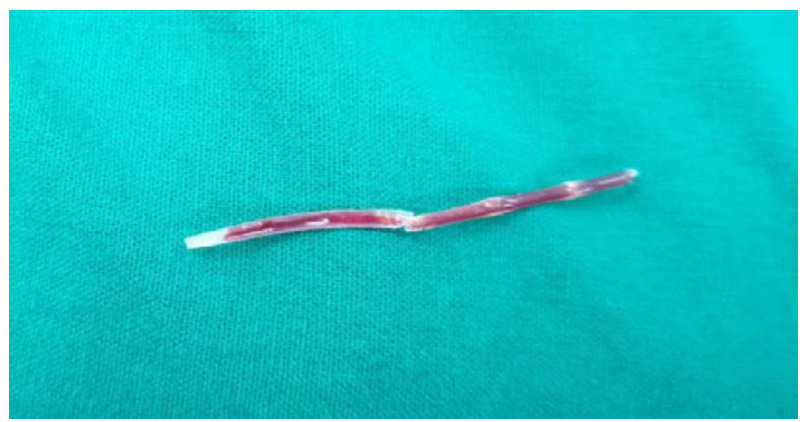

Figure 4. Intravascular portion of the broken cannula.

\section{Discussion}

Peripheral IV cannulation is a common procedure. Approximately $60.0 \%$ of all patients treated by a healthcare system will have an IV cannula inserted for some purpose [1]. Intravenous access is required for blood sampling as well as for the administration of fluids, medications, nutrition and blood products. IV access can be associated with complications such as infiltration to nearby nerves, tendons and blood vessels, thrombophlebitis, venous spasm, haematoma, air embolism and cannula-associated blood stream infections. Among the complications of peripheral venous cannulation, intravascular fracture of the cannula catheter is uncommon. Its exact incidence is unknown, and few studies are available.

The peripheral venous cannula has several components. The catheter part is the intravenous part that is vulnerable to damage or fracture. A broken off piece 
of a cannula within a vein will act as an intravascular foreign body and can embolize. Embolism of broken cannula segments may result in sepsis, endocarditis, cardiac perforations or arrhythmias [2].

Intravenous cannulas can fracture for various reasons. Repeated attempts at insertion, repeated insertion of the introducer needle, a poor-quality cannula or prolonged maintenance of the cannula in situ have been cited as reasons for breakage of these devices. [3]. A partially damaged cannula can completely transect at the time of removal [4].

In the majority oforthopaedic surgery patients, assistive devices (walking frame, crutches) are used to help the patient walk. In most of these patients, peripheral intravenous cannula lines are placed at the dorsum of the hand or wrist, in either the cephalic vein or the basilic vein.

Even though the outer part of the cannula is placed distal to the wrist, the catheter part remains intravenous across the wrist. When a patient mobilizes with a walking aid, the patient's wrist bends to hold the walking aid. When the wrist is bent, the catheter portion of the IV cannula also bends (Figure 1). Due to the inherent flexibility of the catheter part, the cannula bends at the neck of the cannula. Repeated bending of the plastic catheter can result in failure of its integrity and fracture of the catheter part of the cannula. In our patient, obviously, the cannula was placed at the basilic vein at the dorsum of the hand. When the patient held the walking frame, the wrist was bent, and the body weight was loaded at the wrist. It is very obvious that the catheter of the cannula bent at the neck of the cannula. The frequent bending with weight bearing might have resulted in fatigue fracture of the cannula.

In most cases, intravenous fracture of a cannula can be detected at the time of its removal. A nonfunctioning cannula, local swelling and pain at the place of a cannula or symptoms attributable to distal embolization, [3] may be cause for suspicion of cannula fracture; however, fracture may be totally asymptomatic and only discovered at the time of cannula removal [5]. Distal symptoms caused by catheter embolization include cardiac palpitations, dyspnoea, cough, or chest pain [5]. Catheter embolization can also give rise to distal septic complications.

When a cannula fracture is diagnosed, the main priority is to trace the broken part. Attempts to trace along the vein by palpation may dislodge the broken part and facilitate embolization. Applying a tourniquet band proximal to the site can reduce the risk of embolization. However, the application of tourniquets should be limited in duration to prevent tourniquet complications.

Since these devices are made out of a plastic material, they are radiolucent on plain X-ray imaging. Ultrasound scan of the location will help to locate any fragment. If the fragment has migrated to a deeply seated place such as the chest wall or neck, a Computer Tomography (CT) scan may help to locate it [6].

Baliga, K. in his case report about fracture of IV cannula [7], explained inability to locate the cannula part with the xray. He explained the ultrasound guided location and removal with skin incision. He concluded the repeated attempts of 
cannula insertionhad resulted the weakness of the catheter part and fracture of it.

However, Jayanth, B.S. in his case explained the manual palpation along the vein and location of the broken piece and removal [8].

In 1955 Turner, D.D. reported a fatal case of intravenous migration of an IV catheter placed on antecubital vein [9]. It was fractured and migrated. Autopsy revealed an occlusion of right coronary and fatal myocardial infarction.

Even though the fractured IV cannula can migrate to right atrium and there are no reported cases of migration to right atrium.

Glassberg, E., reported a case of peripheral IV cannula fracture during training session due to repeated insertion of the trocar [10].

Currently using peripheral IV cannula (With trocar needle) was introduced around 1957 with widespread use from the beginning of 1970. But there is very few case reports were published in English language literature. This may be due to under reporting of cases.

The simpler procedure of peripheral cannula insertion can result for significant complications. Proper training and technique can prevent potential complications associated with peripheral cannulation. Reintroduction of the needle into a partially inserted cannula should be avoided. Once inserted, the cannula insertion site should be regularly monitored. At removal, the cannula should be checked for completeness. Early recognition of a cannula fracture is prime important. If a piece of the cannula is missing, it is recommended to apply an immediate proximal tourniquet to prevent migration of the missing segment until it is located and removed [5] [6]. Tourniquet application should be performed with caution to prevent complications.

\section{Learning Points}

- At removal, cannulas should be checked for completeness.

- Peripheral venous cannulas should not be placed at the dorsum of the hand or near the wrist joint, especially when the patient has to use walking aids.

- If a piece of a cannula is missing, it is recommended to apply an immediate proximal tourniquet to prevent migration of the missing segment, with precautions for tourniquet complications.

\section{Conclusion}

Peripheral IV cannulation is a safe procedure when it is done with care and precautions. Repeated trocar needle insertion should not be practice during the insertion. It should not place across wrist joint. Integrity of the cannula should check during its usage. At the time of removal complete integrity of the cannula should be confirmed. Health care provider should be well aware about Peripheral IV cannulation risks.

\section{Conflicts of Interest}

The authors declare no conflicts of interest regarding the publication of this paper. 


\section{References}

[1] Lavery, I. and Smith, E. (2007) Peripheral Vascular Access Devices: Risk Prevention and Management. British Journal of Nursing, 16, 1378-1383.

https://doi.org/10.12968/bjon.2007.16.22.27767

[2] Arun, O., Oc, B., Gunduz, E., Oc, M. and Duman, A. (2014) Fracture of an Intravenous Cannula in the Vein Due to Reinsertion of the Guide Needle: A Case Report. Journal-Cardiovascular Surgery, 2, 28-29. https://doi.org/10.5455/jcvs.2014224

[3] Singh, A., Kaur, A., Majhail, S. and Surinder, K. (2015) CT Guided Removal of Iatrogenic Foreign Body: A Broken Intravenous Cannula. Journal of Clinical and Diagnostic Research: JCDR, 9, PD28-PD29. https://doi.org/10.7860/JCDR/2015/14344.6549

[4] Khadim, M.F., Leonard, D., Moorehead, R.A. and Hill, C. (2013) Back to Basics: Iatrogenic Intravenous Cannula Embolus. The Annals of the Royal College of Surge ons of England, 95, e4-e5. https://doi.org/10.1308/003588413X13629960047830

[5] Surov, A., Wienke, A., Carter, J.M., Stoevesandt, D., Behrmann, C., Spielmann, R.P., Werdan, K. and Buerke, M. (2009) Intravascular Embolization of Venous Catheter-Causes, Clinical Signs, and Management: A Systematic Review. Journal of Parenteral and Enteral Nutrition, 33, 677-685. https://doi.org/10.1177/0148607109335121

[6] Goh, Y.H. and Tan, N.G. (2001) Radiological Features of Unusual Ingested Foreign Bodies. Singapore Medical Journal, 42, 129-130.

[7] Baliga, K. and Sujindra, E. (2016) Iatrogenic Complications: A Line's Share. International Surgery Journal, 3, 2250-2253. https://doi.org/10.18203/2349-2902.isj20163040

[8] Jayanth, B.S. and Bhandari, P.M. (2016) Fracture of an Intravenous Cannula in the Vein during Removal: A Case Report. Nitte University Journal of Health Science, 6 , 79-81.

[9] Turner, D.D. and Sommers, S.C. (1954) Accidental Passage of a Polyethylene Catheter from Cubital Vein to Right Atrium: Report of a Fatal Case. New England Journal of Medicine, 251, 744-745. https://doi.org/10.1056/NEJM195410282511806

[10] Glassberg, E., Lending, G., Abbou, B. and Lipsky, A.M. (2013) Something's Missing: Peripheral Intravenous Catheter Fracture. The Journal of the American Board of Family Medicine, 26, 805-806. https://doi.org/10.3122/jabfm.2013.06.130097 